\title{
Minimax Linear Quadratic Gaussian Control of Longitudinal Vibration for Cable Transporter Systems with Multiplicative Nonparametric Uncertainties
}

\author{
Yuhong Zhang \\ Center for Composite Materials, University of Delaware, Newark, DE 19716-3140, USA \\ Sunil K. Agrawal
Department of Mechanical Engineering, University of Delaware, Newark, DE 19716, USA
}

Hemanshu R. Pota and Ian R. Petersen

School of Electrical Engineering, University College, University of New South Wales, ADFA Canberra ACT 2600, Australia

(Received 4 April 2005; accepted 25 July 2005)

\begin{abstract}
This paper demonstrates a new application of robust control to the longitudinal vibration suppression of cable transporter systems with multiplicative nonparametric uncertainties. Both the infinite-dimensional theoretical model for the system and the robust controller are presented. Experimental results have verified the effectiveness of the proposed robust controller. The infinite-dimensional model of the transporter system is truncated to obtain a finite-dimensional model for robust controller design. A minimax Linear Quadratic Gaussian (LQG) controller is designed for multiplicative nonparametric uncertainties. The minimax controller design involves the solution of two simultaneous Riccati equations. The robust controller is implemented on a laboratory cable connected linear transporter system using dSPACE 1103 systems. System identification theory is applied to obtain a statespace representation in the $5-20 \mathrm{~Hz}$ frequency range for the experiment. The experimental results show reductions of $9 \mathrm{~dB}$ in the fundamental resonant peak of the cable transporter system.
\end{abstract}

\section{INTRODUCTION}

Many industrial applications, such as high-rise elevators, ${ }^{1}$ tethered satellite systems, and construction cranes, utilise cables. Modelling and control of vibrations for such cable systems still remains very challenging though extensive studies have been done. Existing techniques to study vibrations include but are not limited to nonlinear control, ${ }^{2,3}$ active vibration control, ${ }^{2,4,5}$ optimisation, ${ }^{6}$ the Lyapunov approach, ${ }^{7}$ and electro-optic holography. ${ }^{8}$ In cable transporter systems, the vibration can be either longitudinal or transverse, or both. The problem of transverse vibration has been considered in reference ${ }^{9,10}$. In this paper, we address the problem of suppression of longitudinal vibrations for a cable-linked system with fixed cable lengths using minimax LQG control theory.

Cable transporter systems usually have infinite-dimensional theoretical models. In this paper, an infinite-dimensional transfer function of the system is first obtained, and then finite-dimensional approximation is applied to obtain a model for controller design. This process necessarily means neglecting unmodelled dynamics in the design of the controller. This is true of control design with finite-dimensional models for all distributed parameter systems. Because of this practical limitation, robust control methods are essential in the design of feedback controllers for distributed parameter systems. In many distributed parameter systems, the collocation of sensors and actuators is used to overcome the spillover effect due to neglected dynamics. ${ }^{11}$ However, in most cable transporter systems, the control actuator is not collo- cated with the transported object. This makes it difficult to use passivity-based control ideas for the design of controllers for cable transporter systems.

We consider robust active control for residual longitudinal vibration suppression of cable transporter systems in this paper. We assume that a feedforward controller is first used to bring the transported object near its final position, ${ }^{\mathbf{1 , 1 2}}$ then a second feedback robust controller is applied to suppress vibrations near this final position. The residual vibrations remain in feedforward control due to the inexact knowledge of the system model in the controller design. Therefore, feedback control is necessary for accurate positioning of the object. We propose a minimax LQG controller to suppress the residual longitudinal vibrations of a cable transporter system. The minimax LQG method minimises a quadratic cost function but with $H_{\infty}$ constrains on the uncertainty. This method has proven very successful in the design of active controllers for the vibration control of a beam ${ }^{13}$ and acoustical noise ${ }^{14}$.

Our main contribution is that we demonstrate the effectiveness of a robust controller for the vibration suppression of a cable transporter system with multiplicative nonparametric uncertainties both theoretically and experimentally. The paper is organised as follows: a dynamic model of the linear transporter system is obtained in Section 2; Section 3 describes the minimax LQG control theory used in this paper; and Section 4 proposes the robust controller design, system identification, uncertainty modelling, and the experimental implementation of the proposed robust controller on a laboratory setup using dSPACE 1103 systems. 